\title{
Ultra-high-speed indirect X-ray imaging system with versatile spatio-temporal sampling capabilities
}

\author{
Emilio M. Escauriza ${ }^{1,2, *}$, Margie P. Olbinado ${ }^{2}$, Michael E. Rutherford ${ }^{1}$, David J. \\ Chapman $^{1}$, John C. Z. Jonsson ${ }^{1}$, Alexander RaCK ${ }^{2}$, And Daniel E. Eakins ${ }^{1}$ \\ ${ }^{1}$ Department of Engineering Science, University of Oxford, Parks Road, Oxford OX1 3PJ, UK \\ ${ }^{2}$ European Synchrotron Radiation Facility, CS 40220, 38043 Grenoble Cedex 9, France \\ *Corresponding author: emilio.escauriza@eng.ox.ac.uk \\ Compiled August 1, 2018
}

A new generation of cameras has made ultra highspeed X-ray imaging at synchrotron light sources a reality, revealing never-before-seen details of sub-surface transient phenomena. We introduce a versatile indirect imaging system capable of capturing - for the first time - hundreds of sequential X-ray pulses in 16bunch mode at the European Synchrotron Radiation Facility, recording at 5.68 Mfps over dozens of microseconds, with an effective exposure of 100 ps. The versatile multiplex camera construction of the system allows for various arrangements, including different scintillator configurations, and simultaneous imaging with different resolutions and regions of interest. Image results from a gas gun impact experiment, in which an additive manufactured aluminium lattice was dynamically compressed, is presented as a demonstration of the system's capabilities.

() 2018 Optical Society of America

OCIS codes: (040.0040) Detectors; (110.0110) Imaging systems; (110.7440) X-ray imaging; (110.4190) Multiple imaging; (320.4240) Nanosecond phenomena; (340.6720) Synchrotron radiation.

http://dx.doi.org/10.1364/ao.XX.XXXXXX

\section{INTRODUCTION}

The demand for ultra high-speed (UHS) X-ray imaging (XRI) has come primarily from research fields that require in-situ X-ray measurements of ultra-fast processes. Here, UHS is defined as recording imaging data at greater than 1 million frames per second (fps) - for a general overview of UHS imaging, refer to [1]. Examples include direct measurements of sub-surface shock dynamics in diamond [2] and high- $Z$ materials, such as additively manufactured (AM) stainless steel lattices [3]; shockwave distributions in granular materials [4]; explosion by plasma expansion during electric arc ignition in a fuse [5]; and crack propagation in glass $[5,6]$. Tracking real-time changes in these experiments requires high temporal and spatial resolutions, as well as a high frame rate to capture the physical and chemical changes that evolve over the ns to ms timescales. A detailed account of UHS XRI and its applications is given by Olbinado et al. [5].

For XRI experiments, there is a choice to be made between direct and indirect detection. Direct detectors offer a comparatively high quantum efficiency, and very low level of noise. However, direct detectors lack versatility and typically have a fixed coarse pixel size [7]. Furthermore, direct detectors are prone to heat and radiation damage from direct exposure in the X-ray beam path. Indirect detection systems offer an alternative solution, by utilising a scintillating screen that re-emits absorbed X-ray photons as visible photons, which are captured by a visible light detector [8]. The optical light path is frequently folded by a mirror away from the $X$-ray beam path toward lenses and a detector, such that they are not directly in the path of the X-rays (see diagram (a) of Fig. 1). The lack of direct X-ray exposure means the electronics of indirect systems avoid radiation damage. Another advantage of indirect detectors is their versatility: different magnifications can be achieved by simply changing the configuration of the optical lenses. Thus, with minimal adjustment, a large range of spatial resolutions can be achieved with a single indirect detector.

The steady pulse of high-intensity $\mathrm{X}$-rays produced by the accelerated electrons in a synchrotron provide an ideal source for performing time-resolved XRI experiments, due to the high brilliance and partial spatial coherence that can be achieved. When operating in one of the so-called 'timing modes', the X-ray pulses are evenly spaced, due to the even distribution of the electrons into tight 'bunches' around the synchrotron's storage ring. This bunching effect is caused by the timing of the energy boost they receive from the radio frequency power supply [9]. The number of electron bunches around the ring is denoted by the 'filling mode'. Current image sensor and scintillator technology allows the $\mathrm{X}$-ray pulses originating from a single bunch to be captured, giving rise to the name 'single-bunch' XRI. The minimum effective exposure of a recorded XRI image is thus determined by the duration of a single $X$-ray pulse, which is given by the bunch duration of $\sim 100 \mathrm{ps}$, and the maximum acquisition frame rate is determined by the separation time between consecutive bunches in a given filling mode (e.g. $153 \mathrm{~ns}$ 
in 24-bunch mode at the Advanced Photon Source (APS) [10] and 176 ns in 16-bunch mode at the European Synchrotron Radiation Facility (ESRF) [11]). Consequently, the maximum frame rate of an XRI image sequence recorded at a synchrotron is equal to the synchrotron's X-ray pulse repetition rate in a given filling mode.

Commercially available cameras used for UHS imaging include those with gated image intensifier charge-coupled device (ICCD) sensors. Such devices are capable of recording scintillator emissions from a single synchrotron $\mathrm{X}$-ray pulse, with very short sensor exposure times ( $<500 \mathrm{ps})$. However, at UHS frame rates, ICCD-based cameras are limited to a two-frame storage limit [12]. Cameras using a complementary metal oxide semiconductor (CMOS) sensor architecture are also capable of single-bunch imaging, but are limited to sub-100 kfps recording [6]. New-generation UHS cameras, which are capable of recording at $>1 \mathrm{Mfps}$ for multiple frames, employ in-situ storage to overcome the limitations of sensor read-out speeds. Using a new-generation UHS camera in combination with 16-bunch filling mode at ESRF beamline ID19, Olbinado et al. performed a single-bunch phase contrast imaging study to capture a $1.9 \mathrm{Mfps}$ sequence of laser-induced shockwave propagation and cavity formation in water [5]. With a similar arrangement, Parab et al. recorded $>1$ Mfps sequences of dynamic fracture in poly(methyl methacrylate) particles at APS beamline 32-ID-B [13].

To capture many sequential X-ray pulses using XRI at a synchrotron facility, a multiplex arrangement is necessary for both gated ICCD and new-generation cameras. Jensen et al. have used a multiplex setup consisting of four gated ICCD cameras each capable of storing two images - to capture up to eight sequential single-bunch frames in 24-bunch filling mode at the APS dynamic compression sector (DCS) beamline 35-ID [14]. Their system also allows the simultaneous imaging of experiments at multiple magnifications, by using different lens configurations on each camera.

In this Letter, we present a UHS XRI system consisting of three CMOS-based new-generation cameras. The system's unprecedented capabilities allow up to 384 single-bunch frames to be captured, with an inter-frame time equal to the inter-bunch time, when using 16-bunch mode at the ESRF. The versatile multiplex construction means it can also be used to simultaneously capture different magnifications and different scintillator emissions. Details of the system implementation and possible configurations are given in section 2 and example XRI images from a gas gun impact experiment of a dynamically compressed AM aluminium lattice are presented in section 3, with conclusions given in section 4 .

\section{INSTRUMENTATION}

In order to capture sequential single-bunch images in 16-bunch mode at the ESRF, a multiplex camera system is used, consisting of three Shimadzu Hyper Vision HPV-X2 cameras. Each camera has a high-speed CMOS burst image sensor, with a $400 \times 250$ pixel array and a $32 \times 32 \mu \mathrm{m}$ pixel size [15]. In the maximum frame rate operation mode, the HPV-X2 camera is fixed to an inter-frame time of $200 \mathrm{~ns}$ ( $5 \mathrm{Mfps}$ ), and in adjustable frame rate mode the minimum inter-frame time is $500 \mathrm{~ns}$ ( $2 \mathrm{Mfps})$, adjustable in $10 \mathrm{~ns}$ increments. The inter-bunch time is $176 \mathrm{~ns}$, meaning synchronisation between the cameras and the bunches is only possible if the inter-frame time for each camera is matched to an integer multiple of the inter-bunch time that is greater than 500 ns. Thus, with three HPV-X2 cameras, it is possible to capture every sequential bunch if each camera
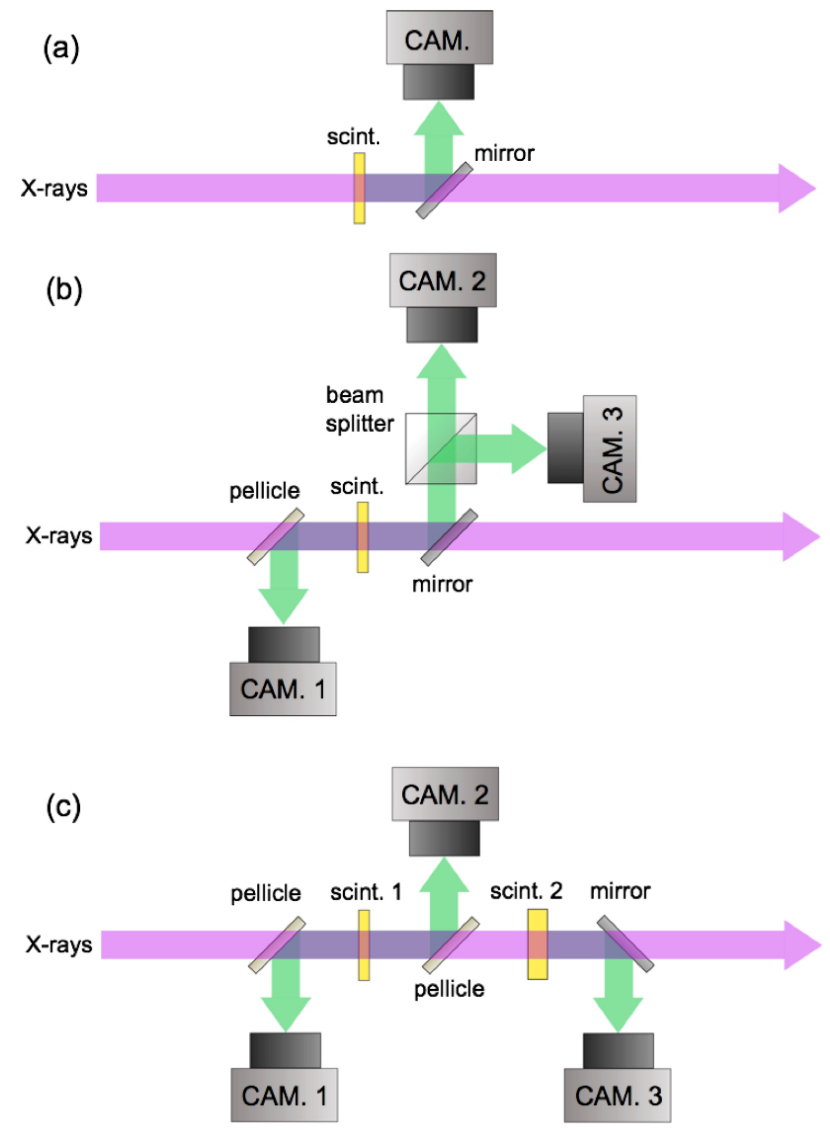

Fig. 1. Diagrams showing three possible camera configurations for performing UHS XRI. (a) A single camera indirectly captures the luminescence from a single scintillator. (b) A three-camera configuration captures the luminescence from a single scintillator. The rear-surface luminescence is split into two optical beams with a 50:50 beam splitter. (c) The configuration used during the experiment presented in this article: a three-camera configuration utilising a $250 \mu \mathrm{m}$ LYSO:Ce scintillator (scint. 1) and a $500 \mu \mathrm{m}$ LYSO:Ce scintillator (scint. 2), placed $100 \mathrm{~mm}$ downstream of the first. The front- and rear-surface luminescence of the upstream scintillator is captured with two cameras via pellicle beam splitters, and the rear-surface luminescence of the downstream scintillator is captured with a third camera via a mirror.

is triggered in sequence, with the inter-frame time set to $530 \mathrm{~ns}$, which is close to the three-bunch separation time of $528 \mathrm{~ns}$. The three sets of images are combined to produce an image sequence with an effective frame rate of $5.68 \mathrm{Mfps}$. A possible arrangement of the three cameras can be seen in diagram (b) of Fig. 1, with the emission from a single scintillator shared between the cameras by exploiting its isotropic luminescence. The upstream scintillator luminescence is folded perpendicular to the X-ray path with a weakly attenuating thin-membrane pellicle beam splitter, toward the first camera (here, we define 'upstream' relative to the direction of $\mathrm{X}$-ray propagation). The downstream luminescence is folded likewise with an optical mirror, then split by a 50:50 beam splitter and captured by the second and third cameras. A consequence of the 50:50 beam splitter arrangement is that the second and third cameras receive less than half of the light compared to the first, meaning signal in those images may be insufficient when imaging materials with higher levels of attenuation.

The arrangement used in the present study can be seen in diagram (c) of Fig. 1. Compared to the arrangement in diagram (b), 
the total light yield is increased by using a second in-line scintillator. For the experiment, two LYSO:Ce (Ce-doped $\mathrm{Lu}_{1.8} \mathrm{Y}_{0.2} \mathrm{SiO}_{5}$, Hilger Crystals, UK) scintillators were used: one of $250 \mu \mathrm{m}$ thickness, and a second of $500 \mu \mathrm{m}$ thickness, placed $100 \mathrm{~mm}$ downstream from the first. LYSO:Ce has a comparatively short characteristic decay time, $\tau \approx 40 \mathrm{~ns}$, making it suitable for imaging high-speed XRI experiments. Other scintillators used for high-speed XRI are GAGG:Ce (Ce-doped $\left.\mathrm{Gd}_{3} \mathrm{Al}_{2} \mathrm{Ga}_{3} \mathrm{O}_{12}\right)$ and YAG:Ce (Ce-doped $\mathrm{Y}_{3} \mathrm{Al}_{5} \mathrm{O}_{12}$ ), with characteristic decay times of $60 \mathrm{~ns}$ and $96 \mathrm{~ns}$, respectively (an overview of scintillator performance in time-resolved XRI is given by Rutherford et al. [16]). The upstream and downstream luminescence of the $250 \mu \mathrm{m}$ scintillator is captured with two cameras, via two Thorlabs BP145B5 1" 300-400 nm pellicle beam splitters, of $5 \mu \mathrm{m}$ thickness, placed in the X-ray beam path. The downstream luminescence of the $500 \mu \mathrm{m}$ scintillator is captured with a third camera via a mirror. Each camera is focused with a motorised 1:1 lens system, consisting of two Nikon f/1.4 (0.36 NA) 50 mm lenses, giving a field-of-view equal to the sensor dimensions of $12.8 \mathrm{~mm} \times$ $8.0 \mathrm{~mm}$.

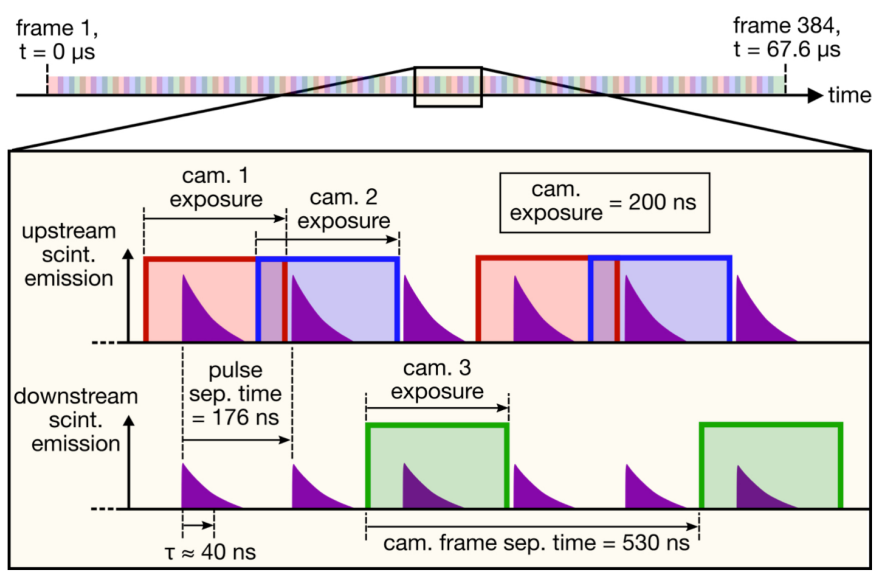

Fig. 2. Diagram showing the timing and synchronisation between the three cameras and the two scintillator emissions. The combined 384frame acquisition lasts for $67.6 \mu \mathrm{s}$, and is represented by the timeline at the top. An expanded section of the timeline shows the exposure times of the three cameras, which are all 200 ns, and how the intensity of the two scintillator emissions varies over time. Cameras 1 and 2 capture the front- and rear-surface emissions of the upstream scintillator, respectively, and camera 3 captures the front-surface emission of the downstream scintillator. The inter-bunch time of $176 \mathrm{~ns}$, inter-frame time of $530 \mathrm{~ns}$ and the LYSO:Ce scintillator decay time of $40 \mathrm{~ns}$ are also shown.

A diagram showing the aforementioned three-camera sequencing, and synchronisation to the emissions of the two scintillators, can be seen in Fig. 2. A single acquisition records 384 frames for a total of $67.6 \mu \mathrm{s}$, with cameras 1 and 2 capturing the luminescence of the upstream scintillator and camera 3 capturing the luminescence of the downstream scintillator. The frame separation time of the cameras is set to $530 \mathrm{~ns}$, such that each camera sequentially captures an image every third X-ray pulse.

The ESRF ID19 X-ray sources used are two U32 undulators operated at minimum gap widths of $11.1 \mathrm{~mm}$ and $11.5 \mathrm{~mm}$, in order to maximise the spectral flux - a requirement for singlebunch XRI, as maximising the signal-to-noise ratio (SNR) is critical at such short exposures. A $1.4 \mathrm{~mm}$ diamond filter minimises the flux of X-rays with energies below $10 \mathrm{keV}$, thus limiting the heat load on the scintillators. The output spectrum after filtering can be seen in the plots (A1) and (B1) of Fig. 3. The mean photon
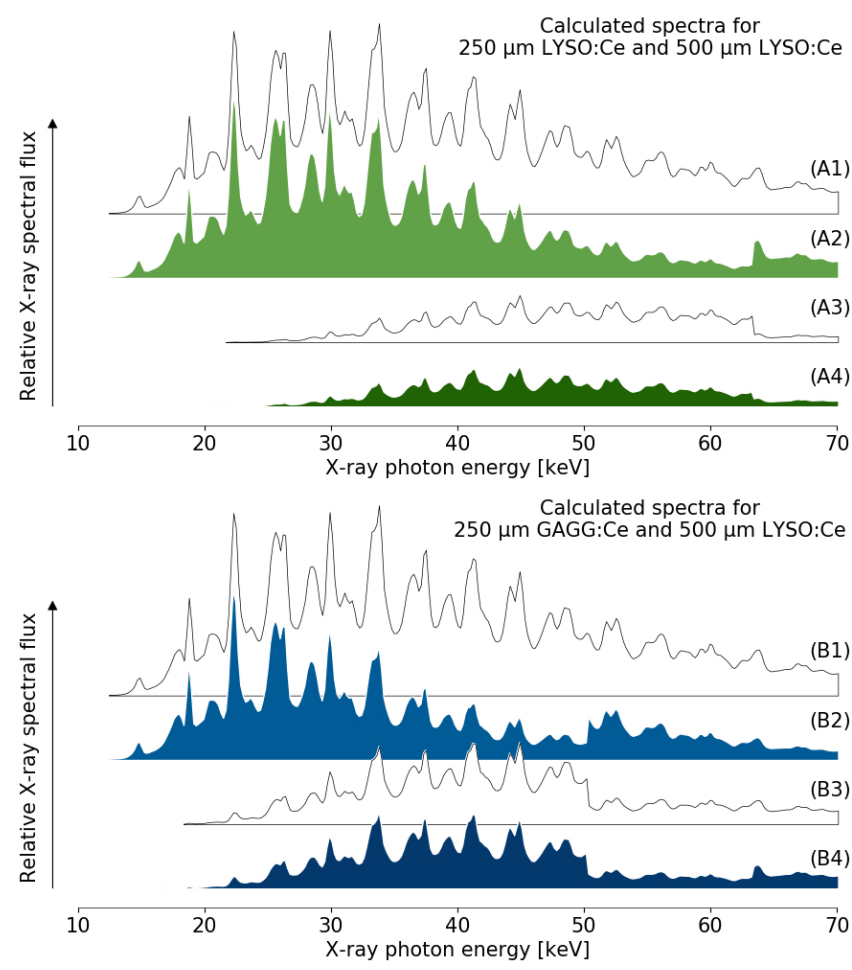

Fig. 3. The predicted photon flux density spectra based on the absorption of filters and the LYSO:Ce and GAGG:Ce scintillators. All calculations have been performed using XOP [17]. Incoming spectra have been represented by a plot outline and absorbed spectra by a solid fill. Spectra $\mathrm{A} 1$ and $\mathrm{B} 1$ show the incoming source after filtering, spectra A2 and $\mathrm{B} 2$ show the absorption of the upstream scintillator, spectra A3 and B3 show the transmission of the upstream scintillator, and spectra A4 and B4 show absorption of the downstream scintillator.

energy is approximately $44 \mathrm{keV}$, with an integral photon flux density (IPFD) of $4.9 \times 10^{15}$ photons $/ \mathrm{mm}^{2} / \mathrm{s}$. The integral photon energy density (IPED) per X-ray pulse is $6.1 \times 10^{-6} \mathrm{~J} / \mathrm{mm}^{2}$ (all calculations performed in XOP [17]).

The absorption and transmission spectra of the upstream $250 \mu \mathrm{m}$ LYSO:Ce scintillator can be seen in plots (A2) and (A3) of Fig. 3, respectively. The mean absorbed energy is around $40 \mathrm{keV}$, and the absorbed IPFD is $3.6 \times 10^{15}$ photons $/ \mathrm{mm}^{2} / \mathrm{s}$, giving an absorbed IPED per pulse of $4.1 \times 10^{-6} \mathrm{~J} / \mathrm{mm}^{2}$. The absorption of the downstream $500 \mu \mathrm{m}$ LYSO:Ce scintillator is shown in plot (A4) of Fig. 3. The mean energy of the downstream scintillator absorption spectrum is higher than for the upstream scintillator, at $55 \mathrm{keV}$. This increase is due to the 'beam hardening' effect, when lower-energy X-rays are absorbed by an object (in this case the upstream scintillator), which increases the mean energy of the transmitted spectrum relative to the incoming spectrum. The absorbed IPFD of the downstream scintillator is $1.0 \times 10^{15}$ photons $/ \mathrm{mm}^{2} / \mathrm{s}$, and the absorbed IPED per pulse is $1.5 \times 10^{-6}$ $\mathrm{J} / \mathrm{mm}^{2}$. The consequences of the different absorption values of the two scintillators, with respect to the recorded XRI images, will be discussed in the following section.

\section{ULTRA HIGH-SPEED RADIOGRAPHY}

The experiment, consisting of an impact target and gas gun projectile launcher, is placed approximately $150 \mathrm{~m}$ downstream from the source, with the impact direction perpendicular to the X-ray beam. The detector is placed a further $7 \mathrm{~m}$ down- 
stream from the target, ensuring the recorded images are in the propagation-based 'phase contrast' regime - interference between the X-rays after they pass through the target material increases the contrast of material edges and interfaces [18, 19]. As the $\mathrm{X}$-rays propagate into the first Fresnel diffraction zone, interference fringes form, with a characteristic length given by $\sqrt{\lambda z} \approx 14 \mu \mathrm{m}$ (where $\lambda=0.28 \AA$ is the mean X-ray wavelength and $z=7 \mathrm{~m}$ is the target-to-detector distance). Therefore, with a pixel size of $32 \mu \mathrm{m}$, and with the main features of the object being significantly larger than the characteristic length, the edge contrast in the recorded images is enhanced, while preserving the attenuation projection of the target.

The example application shown in this Letter is a dynamic compression experiment, in which an AM aluminium lattice was impacted with a gas gun projectile. Such an experiment typically involves in-situ optical and surface measurements, and ex-situ analysis of the target after impact. Synchrotron UHS XRI systems now enable the in-situ tracking of subsurface phenomena, with a 100 ps effective exposure per image. Hawreliak et al. [20] and Branch et al. [21] performed UHS XRI impact studies on AM polymer lattices at APS, capturing up to eight frames per impact. Our three-camera system extends the capabilities of UHS XRI, with each camera capturing up to 128 frames per experiment. The combined 384-frame image sequence has an inter-frame time of $176 \mathrm{~ns}$ (equivalent to $5.68 \mathrm{Mfps}$ ), allowing the tracking of phenomena as they evolve over dozens of $\mu$ s. As well as gas gun impact studies, other applications for this versatile imaging system include laser shock [22], crack propagation [5, 6] and laser powder bed fusion [23].

Single-bunch radiographs from the experiment can be seen in Fig. 4 and Fig. 5, taken from a 384-frame, 5.68 Mfps image sequence. The images have been normalised to highlight features, meaning grey levels should not be taken to represent absolute intensity. Phase-contrast is evident in the images as bright fringes at material interfaces. The time stamps are relative to the first image, and are separated by the inter-bunch time of 176 ns. Fig. 4 shows the details of the impact: the lattice target, which is shown stationary in frame 1 , is built onto a $2 \mathrm{~mm}$ thick aluminium plate. The target is impacted from the left with a $3 \mathrm{~mm}$ thick aluminium flyer plate at $160 \mathrm{~m} / \mathrm{s}$. Deformation at a later time can be seen in frame 199. Changes between the images evolve slowly, with the fastest details in the material crossing approximately one of the $32 \mu \mathrm{m}$ pixels every image (equivalent to a velocity of $180 \mathrm{~m} / \mathrm{s}$ ).

Recalling diagram (c) of Fig. 1, Fig. 5 shows three sequential radiographs, each captured with a different camera. Frames 67 and 68 , captured with cameras 1 and 2 via the thinner upstream scintillator, show similar levels of contrast and signal. The contrast in frame 68 is approximately twice the contrast in frame 69, which was captured with camera 3 via the thicker downstream scintillator. This is due to the beam hardening effect discussed in the previous section. The X-ray spectrum that reaches the second scintillator experiences lower attenuation from the target material, because of its higher average energy. Thus, the contrast between the sample and the unattenuated sections is lower in the resulting images. Furthermore, the noise level in frame 69 is higher than in frames 67 and 68 , due to a $63 \%$ decrease in the absorbed energy of the downstream scintillator relative to the upstream scintillator. This is represented in the images captured via the different scintillators - the average pixel count of frame 69 is $\sim 64 \%$ less than in frame 68 . According to the statistics of the Poisson noise in the images, the SNR scales with $\sqrt{N}$, where
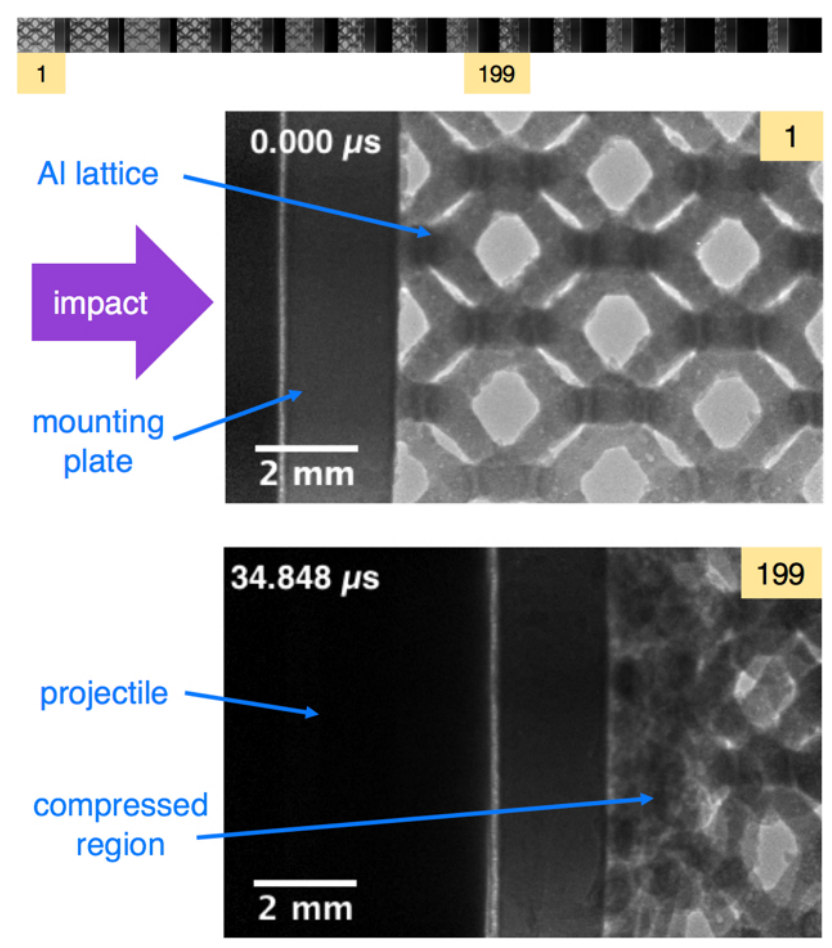

Fig. 4. Single-bunch radiographs of the gas gun impact experiment, performed in 16-bunch mode at the ESRF. The images are taken from a 384-frame, 5.68 Mfps image sequence. The top row of smaller images are separated by 22 frames, starting from frame 1 . The larger images show frames 1 and 199. Frame 1 shows the details of the target: an AM aluminium lattice built onto a $2 \mathrm{~mm}$ thick aluminium plate is impacted from the left at $160 \mathrm{~m} / \mathrm{s}$ by a projectile tipped with a $3 \mathrm{~mm}$ thick aluminium flyer plate. Frame 199 shows the details of the impact: compression of the lattice is visible in the right of the images, and the darkened region on the left is the highly-attenuating projectile.

$N$ is the number of visible photons detected (this scales with the total energy of X-ray photons absorbed by the scintillator). Our SNR measurements confirm this to be the case, with a $\sim 43 \%$ decrease in SNR when comparing frame 68 to frame 69. Despite this drop, the SNR in frame 69 is sufficient to distinguish features in the radiographs. However, for thicker samples or high-Z materials, transmission through the sample will likely not be high enough to produce sufficient signal.

The two-scintillator multiplex arrangement has the advantage of fully utilising the X-ray flux, with $\sim 75 \%$ of the spectrum first attenuated by the $250 \mu \mathrm{m}$ scintillator, after which $\sim 80 \%$ of the remaining, harder spectrum is attenuated by the $500 \mu \mathrm{m}$ scintillator. This results in $\sim 95 \%$ total X-ray absorption. Furthermore, the pellicle beam splitters allow the isotropic luminescence of the scintillators to be exploited without further attenuating the X-rays, thus maximising the total signal yield for each image. This setup is therefore ideal for single-bunch imaging of ultra-fast processes when the maximum light yield is desired. Diagram (b) of Fig. 1, where every camera captures the emission from a single scintillator, would be suitable for experiments where it is required to have the same incoming X-ray spectrum for each image. This setup would also be suitable in experiments for which light yield is not a concern, e.g. for weakly attenuating samples or for longer exposures.

With both the two-scintillator and single-scintillator arrangements, it is possible to incorporate a fourth camera. In the former case, by capturing the front-surface luminescence of the second 

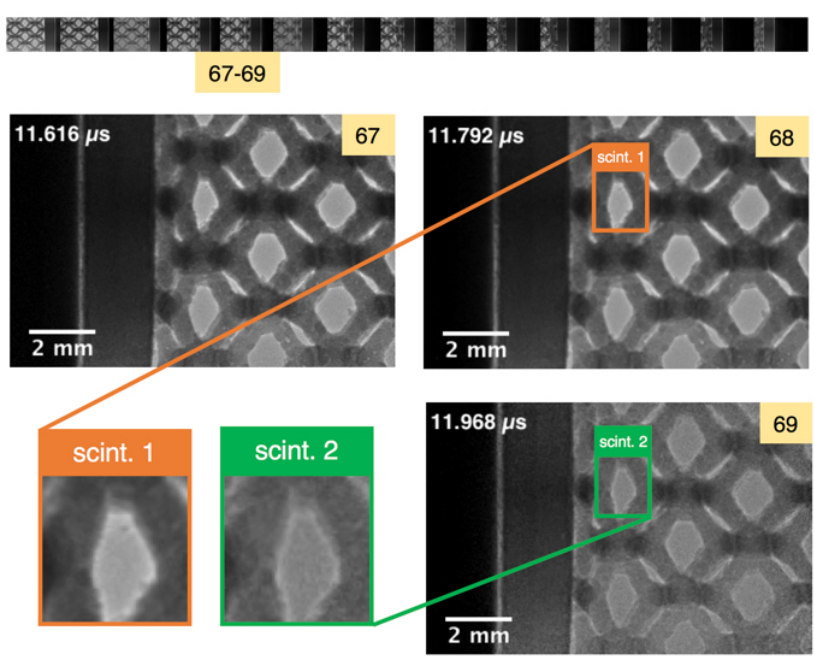

Fig. 5. Single-bunch radiographs from the experiment. The top row of smaller images are separated by 22 frames, starting from frame 1 . The larger images show frames 67-69. The inter-frame time is equal to the inter-bunch time of $176 \mathrm{~ns}$. Frames 67 and 68 were captured by cameras 1 and 2, respectively, via the upstream $250 \mu \mathrm{m}$ scintillator. Frame 69 was captured with camera 3 via the downstream $500 \mu \mathrm{m}$ scintillator. Sections from frame 68 and 69 have been enlarged to highlight the difference in contrast from the two scintillators.

scintillator, and in the latter case, by splitting the upstream emission with a 50:50 beam splitter. In principle, it is also possible to incorporate more cameras, through further splitting of the optical beams, as demonstrated by Jensen et al. [14], with their four-camera, gated ICCD arrangement. They also showed an additional capability of a multiplex imaging system: the magnification of each camera can be customised by altering its lens configuration, allowing simultaneous UHS XRI of processes with multiple resolutions and regions of interest. This 'hierarchical' approach has also been demonstrated by Rack et al. [24].

The plots (B1)-(B4) of Fig. 3 show the predicted spectra for an alternative scintillator arrangement, in which a $250 \mu \mathrm{m}$ GAGG:Ce scintillator replaces the $250 \mu \mathrm{m}$ LYSO:Ce upstream scintillator. The IPED absorbed by GAGG:Ce per pulse is $\sim 26 \%$ less than LYSO:Ce, and the K-edge positions for GAGG:Ce and LYSO:Ce are $50 \mathrm{keV}$ and $64 \mathrm{keV}$, respectively. These two factors result in an approximate $\sim 65 \%$ increase in photon energy absorption of the downstream scintillator for the GAGG-LYSO setup, when compared to the LYSO-LYSO setup used during the experiment. Additionally, the difference in absorbed IPED per pulse of the two scintillators is smaller for the new setup, with the upstream scintillator absorbing $3.0 \times 10^{-6} \mathrm{~J} / \mathrm{mm}^{2}$ and the downstream scintillator absorbing $2.5 \times 10^{-6} \mathrm{~J} / \mathrm{mm}^{2}$. From the $\sim 18 \%$ decrease in signal that this expected to produce (relative to the upstream scintillator), we predict that the decrease in SNR will be $\sim 9 \%$ for the GAGG-LYSO setup. This is a significant improvement when compared to the signal and SNR differences of $\sim 64 \%$ and $\sim 43 \%$, respectively, for the LYSO-LYSO setup used in the experiment.

Furthermore, we predict that the difference in contrast between the images obtained on the different scintillators will be smaller, because the predicted mean energy absorption between the two scintillators in the GAGG-LYSO setup is less, at $11 \mathrm{keV}$, compared to $15 \mathrm{keV}$ in the LYSO-LYSO case. The improvements in SNR difference and contrast will likely result in combined radiograph sequences with a more uniform image response, which is key in experiments that require higher levels of consistency between the images, such as when looking at thicker samples with lower signal. It should be noted, however, that in the images captured via the GAGG:Ce scintillator, there is the potential introduction of ghosting effects. This would be due to the longer characteristic decay time of the GAGG:Ce luminescence, when compared to LYSO:Ce [16].

Another potential advantage of a two in-line scintillator arrangement is the hardened X-ray spectrum observed by the second scintillator, which could also be used in studying composite materials with high- $Z$ inclusions. Different scintillator combinations may be used to increase this difference. With the lower-energy portion of the transmitted spectrum removed by the first scintillator, higher levels of transmission through high- $Z$ regions of the sample will be visible in the images recorded via the second scintillator. Using two scintillators of varying composition also presents the opportunity to generate two images of a dynamic event simultaneously, with different absorption characteristics. For experiments involving attenuation measurements, such as density calculations, this arrangement would increase the amount of information recorded per event.

In the experiment presented, the extended recording time of the imaging system has been demonstrated, with the impact spanning the majority of the frames. The extended recording capability will also be beneficial when investigating the onset of rapid $(<1 \mu \mathrm{s})$ failure processes in brittle materials, such as the fracture of ceramics [25] and adiabatic shear band formation [26], where, due to the complexity of the system the impact-tofailure time may be uncertain. In such cases, a full 384-frame recording can be made, centred on the predicted event time, thus increasing the probability of capturing the event.

However, the $5.68 \mathrm{MHz}$ frame rate capability is not fully demonstrated by the presented experiment, since only small changes are visible between frames. Experiments involving events with faster time evolution will exploit the high frame rate, when it is crucial to use a single bunch per image, because of blurring that occurs if two X-ray pulses are captured per camera exposure (e.g. blurring of size $176 \mu \mathrm{m}$ will occur for an object travelling at $1 \mathrm{~km} / \mathrm{s}$ ). Examples of faster processes that could be imaged with this system include crack wave propagation in silicon [27] and supersonic jet formation in aluminium [28]. Though, as the speed of objects imaged increases, the portion of the frames providing data will decrease (e.g. an object travelling at $2 \mathrm{~km} / \mathrm{s}$ will cross the field of view in 34 frames). Future advances in scintillator and detector technology may allow single-bunch imaging in 'uniform' fill mode at the ESRF, which operates with 992 bunches and an inter-bunch time of $2.8 \mathrm{~ns}$. This would give a frame rate of $352 \mathrm{MHz}$, and allow for the extended recording of objects travelling many $\mathrm{km} / \mathrm{s}$.

For synchrotron imaging, vast improvements in resolution and frame rate can be made before the limit of the 100 ps effective exposure is reached. This is because, at present, there is a three orders of magnitude difference between the $176 \mathrm{~ns}$ interbunch time and the 100 ps effective exposure. For example, the detector resolution would have to approach $1 \mu \mathrm{m}$ for blurring to be significant, if imaging an object moving at $10 \mathrm{~km} / \mathrm{s}$. It is also worth mentioning that, with a frame rate of $5.68 \mathrm{MHz}$, the system is also compatible with the timing of the bunch-trains at the European XFEL (Hamburg, Germany) where fs-short X-ray pulses are available at a frequency of $4.5 \mathrm{MHz}$ [29]. 


\section{CONCLUSIONS}

We have demonstrated a newly-developed indirect UHS XRI system with a highly versatile multiplex construction. With this imaging system, it is now possible to capture sequential bunches over extended periods in 16-bunch mode at the ESRF. The threecamera, two-scintillator setup is capable of producing image sequences at $5.68 \mathrm{Mfps}$ for $67.6 \mu \mathrm{s}$, recording a maximum of 384 frames. As a proof-of-concept, results from a gas gun experiment using the imaging system have been presented, in which an AM aluminium lattice was dynamically compressed. The limitations of the system and the scope for future improvements in synchrotron UHS XRI have been considered in the context of the image results.

The pros and cons of the two-scintillator setup have been discussed, with comparisons between the radiographs from each scintillator. Despite a reduction in SNR and contrast in the images recorded from the second scintillator, the values were still sufficient to provide data on the compression dynamics of the lattice structure. To address the issue in contrast from the second scintillator in the LYSO-LYSO setup, an alternate GAGG-LYSO set-up was also discussed, with the predicted spectra showing closer levels of absorption between the two scintillators, which we expect will produce more uniformity between the images. This setup could also be used to simultaneously obtain image data from multiple spectra, a useful capability for studying heterogeneous samples.

Looking toward the future of UHS XRI, the 'Extremely Brilliant Source' (EBS) upgrade to the ESRF will provide orders of magnitude increases in X-ray flux density, thus increasing the range of configurations for this imaging system, beyond those already mentioned, by allowing for the scintillator emission to be further split and more cameras incorporated [30]. These developments will greatly increase the range of experimental applications for this system.

The imaging system was developed as part of the ESRF Long Term Proposal MI-1252.

PhD funding support provided by the ESRF and First Light Fusion Ltd., and the continued support from DSTL, EPSRC, AWE, and the Institute of Shock Physics (Imperial College London), is gratefully acknowledged.

In particular, the authors would like to thank: Martin Jäcklein and Aron Pfaff (Fraunhofer EMI) for providing the aluminium lattice target used in the experiment; Brian Speyer (Speyer Photonics Ltd) and Hugo Doyle (First Light Fusion Ltd.) for each loaning a Shimadzu HPV-X2 camera for the experiment; Benoît Roche for providing bunch current data; Paul Tafforeau (ESRF beamline ID19) for advice on the spectrum calculations; David Paganin for comments on versions of the manuscript; and David Pitman and Robert Denning (Imperial College London) for their help with preparations and invaluable support during the beam time.

\section{REFERENCES}

1. K. Tsuji, "History of Curiosity," in "The Micro-World Observed by Ultra High-Speed Cameras," K. Tsuji, ed. (Springer, Cham, 2018), chap. 1, pp. 3-5, 1st ed.

2. A. Schropp, R. Hoppe, V. Meier, J. Patommel, F. Seiboth, Y. Ping, D. G. Hicks, M. A. Beckwith, G. W. Collins, A. Higginbotham, J. S. Wark, H. J. Lee, B. Nagler, E. C. Galtier, B. Arnold, U. Zastrau, J. B. Hastings, and C. G. Schroer, "Imaging Shock Waves in Diamond with Both High
Temporal and Spatial Resolution at an XFEL," Scientific Reports 5, 1-8 (2015).

3. D. E. Eakins and D. J. Chapman, "X-ray imaging of subsurface dynamics in high- $Z$ materials at the Diamond Light Source," Review of Scientific Instruments 85 (2014).

4. M. E. Rutherford, D. J. Chapman, J. G. Derrick, J. R. W. Patten, P. A. Bland, A. Rack, G. S. Collins, and D. E. Eakins, "Probing the early stages of shock-induced chondritic meteorite formation at the mesoscale," Scientific Reports 7 (2017).

5. M. P. Olbinado, X. Just, J.-L. Gelet, P. Lhuissier, M. Scheel, P. Vagovic, T. Sato, R. Graceffa, J. Schulz, A. Mancuso, J. Morse, and A. Rack, "MHz frame rate hard $\mathrm{X}$-ray phase-contrast imaging using synchrotron radiation," Optics Express 25 (2017).

6. A. Rack, M. Scheel, L. Hardy, C. Curfs, A. Bonnin, and H. Reichert, "Exploiting coherence for real-time studies by single-bunch imaging," Journal of Synchrotron Radiation 21, 815-818 (2014).

7. T. Hatsui and H. Graafsma, "X-ray imaging detectors for synchrotron and XFEL sources," IUCrJ 2, 371-383 (2015).

8. S. M. Gruner, M. W. Tate, and E. F. Eikenberry, "Charge-coupled device area x-ray detectors," Review of Scientific Instruments 73, 2815-6023 (2002).

9. P. Willmott, "Synchrotron Physics," in "An Introduction to Synchrotron Radiation," (John Wiley \& Sons, Ltd, 2011), chap. 3, pp. 39-86.

10. A. Miceli, "Application of Pixel array detectors at X-ray synchrotrons," Journal of Instrumentation 4 (2009).

11. M. Wulff, F. Schotte, G. Naylor, D. Bourgeois, K. Moffatb, and G. Mourouc, "Time-resolved structures of macromolecules at the ESRF: Single-pulse Laue diffraction, stroboscopic data collection and femtosecond flash photolysis," Nuclear Instrurnents and Methods in Physics Research A 398, 69-84 (1997).

12. "PI-MAX4:1024i Review P1," Tech. rep.

13. N. D. Parab, Z. Guo, M. C. Hudspeth, B. J. Claus, K. Fezzaa, T. Sun, and W. W. Chen, "Dynamic fracture behavior of single and contacting Poly(methyl methacrylate) particles," Advanced Powder Technology 28, 2929-2939 (2017).

14. B. J. Jensen, "LANL DCS Activities, Highlights and Perspectives," in "DOE/NNSA DCS Review September 20-21, 2016," (2016), pp. 1-23.

15. Y. Tochigi, K. Hanzawa, Y. Kato, R. Kuroda, H. Mutoh, R. Hirose, H. Tominaga, K. Takubo, Y. Kondo, and S. Sugawa, "A Global-Shutter CMOS Image Sensor with Readout Speed of 1Tpixel / s Burst and 780Mpixel / s Continuous," IEEE Journal of Solid-State Circuits 48, 329-337 (2013)

16. M. E. Rutherford, D. J. Chapman, T. G. White, M. Drakopoulos, A. Rack, and D. E. Eakins, "Evaluating scintillator performance in time-resolved hard X-ray studies at synchrotron light sources," Journal of Synchrotron Radiation 23, 685-693 (2016).

17. M. Sánchez del Río and R. J. Dejus, "XOP 2.1 - A New Version of the X-ray Optics Software Toolkit," AIP Conference Proceedings 705, 784-787 (2004).

18. S. W. Wilkins, T. E. Gureyev, D. Gao, A. Pogany, and A. W. Stevenson, "Phase-contrast imaging using polychromatic hard X-rays," Nature 384, 335-338 (1996).

19. P. Cloetens, R. Barrett, J. Baruchel, J.-P. J.-P. Guigay, and M. Schlenker, "Viewpoint on 'Phase objects in synchrotron radiation hard x-ray imaging'," Journal of Physics D: Applied Physics 29, 133-146 (1996).

20. J. A. Hawreliak, J. Lind, B. Maddox, M. Barham, M. Messner, N. Barton, B. J. Jensen, and M. Kumar, "Dynamic Behavior of Engineered Lattice Materials," Scientific Reports 6 (2016).

21. B. Branch, A. Ionita, B. E. Clements, D. S. Montgomery, B. J. Jensen, B. Patterson, A. Schmalzer, A. Mueller, and D. M. Dattelbaum, "Controlling shockwave dynamics using architecture in periodic porous materials," Journal of Applied Physics 121 (2017).

22. M. P. Olbinado, V. Cantelli, O. Mathon, S. Pascarelli, J. Grenzer, A. Pelka, M. Roedel, I. Prencipe, A. L. Garcia, U. Helbig, D. Kraus, U. Schramm, T. Cowan, M. Scheel, P. Pradel, T. De Resseguier, and A. Rack, "Ultra high-speed x-ray imaging of laser-driven shock compression using synchrotron light," Journal of Physics D: Applied Physics 51, 1-11 (2018). 
23. C. Zhao, K. Fezzaa, R. W. Cunningham, H. Wen, F. De Carlo, L. Chen, A. D. Rollett, and T. Sun, "Real-time monitoring of laser powder bed fusion process using high-speed X-ray imaging and diffraction," Scientific Reports 7 (2017).

24. A. Rack, F. García-Moreno, L. Helfen, M. Mukherjee, C. Jiménez, T. Rack, P. Cloetens, and J. Banhart, "Hierarchical radioscopy using polychromatic and partially coherent hard synchrotron radiation." Applied Optics 52, 8122-7 (2013).

25. T. Camacho and M. Ortizt, "Computational Modelling of Impact Damage in Brittle Materials," International Journal of Solids and Structures 33, 2899-2938 (1996).

26. Z. Zhang, D. E. Eakins, and F. P. E. Dunne, "On the formation of adiabatic shear bands in textured HCP polycrystals," International Journal of Plasticity 79, 196-216 (2016).

27. D. Massy, F. Mazen, S. Tardif, J. D. Penot, J. Ragani, F. Madeira, D. Landru, O. Kononchuk, and F. Rieutord, "Fracture dynamics in implanted silicon," Applied Physics Letters 107 (2015).

28. J. M. Foster, B. H. Wilde, P. A. Rosen, T. S. Perry, M. Fell, M. J. Edwards, B. F. Lasinski, R. E. Turner, and M. L. Gittings, "Supersonic jet and shock interactions," Physics of Plasmas 9, 2251-2263 (2002).

29. A. Aghababyan, C. Bohm, P. Gessler, A. Hidvégi, H. Kay, G. Petrosyan, L. Petrosyan, V. Petrosyan, and K. Rehlich, "XFEL Timing System Specifications Version 2.2," Tech. rep., Hamburg (2013).

30. R. Dimper, H. Reichert, P. Raimondi, L. Sánchez Ortiz, F. Sette, and J. Susini, The Orange Book: ESRF Upgrade Programme Phase II (2015-2022) (2015). 